\title{
Trabajo comunitario en el recurso turístico natural cascada Pailón San Jacinto, Parroquia Febres Cordero, Cantón Babahoyo
}

\section{Community work in the natural tourist resort Pailón San Jacinto waterfall, Parroquia Febres Cordero, Cantón Babahoyo.}

\author{
Christian Rivera García. ${ }^{1}$, Darío Arellano Valencia. ${ }^{2}$, \& Stephany Hurtado. ${ }^{3}$
}

\begin{abstract}
.
In the present investigation it is tried to generate knowledge referring to the tourist activity that is developed in the province of the Rivers, is necessary to consider antecedents that were creating step by step the tourist phenomenon of which nowadays we speak. The clear differences between the socio-economic and cultural benefits granted by tourism compared to other activities of daily living, it is important to define the resources and elements involved in the tourism product to be offered, in order to motivate the displacement and meet the needs of the traveler, meeting the expectations of the visitor and their experience in determining their expectations. It is essential to identify the motivations that allow the tourist to travel to the different destinations programmed in the province, to direct the visit from curiosity and recreation, with a crystalline attachment to conservation, protection and prevention of natural resources undertaking appropriate planning of the use of landscape resources, flora and fauna that make up different ecosystems of great value.
\end{abstract}

Keywords: Community work, natural tourism resource, water resource, tourism potential.

\footnotetext{
${ }^{1}$ Universidad Técnica de Babahoyo, Ecuador, crivera@utb.edu.ec

${ }^{2}$ Universidad Técnica de Babahoyo, Ecuador, darellano@utb.edu.ec

${ }^{3}$ Universidad Técnica de Babahoyo, Ecuador, stefanie11021@ gmail.com
} 


\section{Resumen.}

En la presente investigación se pretende generar conocimiento referente a la actividad turística que se desarrolla en la provincia de Los Ríos, es necesario considerar antecedentes que fueron creando paso a paso el fenómeno turístico del que hoy en día hablamos. Las claras diferencias entre los beneficios socio-económicos y culturales que otorga el turismo en comparación con otras actividades del diario vivir, es importante definir los recursos y elementos involucrados en el producto turístico a ofertar, a fin de motivar el desplazamiento y satisfacer las necesidades del viajero, cumpliendo con las expectativas del visitante y su experiencia determinante en sus expectativas. Es imprescindible identificar las motivaciones que permiten el desplazamiento del turista hacia los diferentes destinos programados en la provincia, para encaminar la visita desde la curiosidad y la recreación, con un cristalino apego a la conservación, protección y prevención de los recursos naturales emprendiendo una planificación adecuada hacia el aprovechamiento racional de los recursos paisajísticos, flora y fauna que componen diferentes ecosistemas de gran valor.

Palabras claves: Comunicación integrada de marketing, público objetivo, empresa.

\section{Introducción.}

Ecuador desde hace algún tiempo emplea la palabra turismo en los sectores rurales, teniendo como finalidad el turismo comunitario, mostrando cambios socioeconómicos significativos. En la provincia de Los Ríos no se ha desarrollado formalmente el turismo comunitario sin embargo existen asociaciones agrícolas que están emprendiendo la actividad turística como fuente económica. Esta investigación se basó en analizar el trabajo comunitario para el aprovechamiento del recurso turístico natural cascada pailón San Jacinto con el propósito de ofrecer mejor calidad de vida para los habitantes, fortalecer la identidad cultural e implementar programas de manejo racional de los recursos hídricos de la parroquia Febres Cordero del cantón Babahoyo.

Trabajar en el desarrollo comunitario involucra directamente a agrupaciones o asociaciones jurídicamente establecidas, encaminadas al trabajo sistemático en infraestructura turística, creación y desarrollo de nuevas fuentes de ingresos, en la promoción de la vida social y cultural, en definitiva, participar en la vida cotidiana de una comunidad diferente de la nuestra, es una oportunidad de experimentar rutinas nuevas e intensas. 
En la presente investigación, se identificaron algunas causas que han ocasionado el problema de investigación, conjuntamente con las variables de atención y soporte para la información basadas en los niveles, macro, meso y micro, desvirtuando unidades de observación anidadas en la invisibilidad de planificación, puntualizando la minimización de impactos negativos que ocasionan diversos problemas en el recurso turístico natural responsabilidad de la comunidad en el aprovechamiento de la riqueza natural y cultural.

En la Unión Europa atendiendo al trabajo comunitario, se creó la Ruta del Vino es una iniciativa comunitaria turística en demanda relativamente estable, en 2016. En Europa existen 75 millones de viajeros al año interesados en experimentar las actividades turísticas en referencia al turismo comunitario, que buscan experimentar de forma más auténtica las culturas y recursos turísticos naturales locales y favorecer al desarrollo sostenible desde perspectivas económicas, sociales y medioambientales. (Escolar, B. M., \& Morueco, R. F. 2011).

El continente Asiático implementa medidas sobre los recursos turísticos naturales para su mejoramiento, la gestión ambiental y el desarrollo turístico. Además el trabajo comunitario se relaciona con la parte turística donde pobladores se reúnen para establecer ciertas características, aspectos y actividades que podrían ayudar en la promoción de un recurso turístico. (Fayos-Solá, E. 2004).

En África el gobierno creó programas de trabajos comunitarios para lograr detener la pobreza, desempleos y desigualdad, dando oportunidades a los pobladores a realizar actividades durante ocho días al mes, para los participantes no es remunerable la situación, pero para el gobierno es la mejor manera en lidiar con la escases de alimentos básicos en algunas familias. (Díaz, D. C., Hernández, I. P., \& Díaz, J. P. C. 2017).

En África Occidental y Oriental, la "Perla de África" se encuentra una gran biodiversidad, recursos naturales, diferentes tipos de flora fauna y tribus las cuales se dedican al trabajo comunitario, teniendo como instrumento principal el turismo. Para llamar la atención del turista se valen de su riqueza cultural y natural método fundamental en el crecimiento económico. (Díaz, D. C., Hernández, I. P., \& Díaz, J. P. C. 2017).

En América Latina se creó la Red de Turismo Comunitario con el propósito de que exista equidad social, identidad cultural y preservación de los recursos naturales, está integrada por red de comunidades campesinas e indígenas, instituciones de apoyo que comparten un enfoque sobre el desarrollo sostenible del turismo. (REDTURS, 2007).

El objetivo principal de REDTURS es promover oportunidades de empleo a las comunidades de América Latina mejorando sus condiciones de vida y empleo, logrando alcanzar la estrategia del Sumak kawsay (Buen Vivir), como son: erradicar la pobreza extrema, promover la igualdad entre los géneros y garantizar la sostenibilidad del medio ambiente. (REDTURS, 2007). 
Por otra parte el trabajo comunitario en Venezuela, Chile, Bolivia inició proceso de innovación urbana que se trata de capacitar a la comunidad con el fin de que estas gestionen sus propios proyectos de servicio público turísticos e infraestructura turística, con el objetivo de satisfacer necesidades y expectativas de calidad de vida y desarrollo humano. (Pochat, V., Donoso, M., \& Saldarriaga, J. 2018).

En Ecuador hay más de 52 destinos dedicados al trabajo comunitario con una orientación participativa en la actividad turística de la zona. Ecuador es uno de los países más desarrollado y reconocido por la práctica del turismo comunitario, con la ayuda de la autogestión de las comunidades, conjuntamente con el aporte que se ha recibido de las agencias de cooperación internacional y organizaciones no gubernamentales sin fines de lucro. (REDTURS, 2007). Ecuador está ubicado en la región Noroccidental de América del sur, mega diverso en todo su esplendor, cuenta con veinticuatro provincias divididas en cuatro regiones Andina, Amazónica, Litoral e Insular. A fines de los años 90 surge la necesidad y el derecho de reconocimiento y legalización de la actividad comunitaria en el turismo del cual surge la creación de la Federación Plurinacional de Turismo Comunitario del Ecuador (FEPTCE), organización que permite los derechos culturales de los pueblos y nacionalidades del Ecuador, "Turismo comunitario es toda actividad turística solidaria que permite la participación activa de la comunidad desde una perspectiva intercultural, manejo adecuado del patrimonio cultural, basado en un principio de equidad en la distribución de los beneficios locales" (FEPTCE, 2006). La pluriculturalidad cuenta con diferentes etnias indígenas, los más comunes son Shuar, Afroecuatorianos, Salasacas, Manteños, Saraguros, entre otros, indígenas, pertenecientes a sectores de atención prioritaria inspiración de nuevos proyectos para el desarrollo económico de un país aventurero, que en la actualidad han ido desarrollándose con ideas proactivas como es el caso del turismo comunitario (Solís Ruiz, M. A. 2016).

Sus principales trabajos en el turismo comunitario, son la elaboración de artesanías fabricadas con materia prima de la región y actividades turísticas en base a las cuatro necesidades del turista: alimentación, hospedaje, transporte e información turística. (Solís Ruiz, M. A. 2016).

"Cacha", es una de las nacionalidades indígenas del Ecuador donde se asentaron los Puruhuaes, ancestro de Atahualpa, Rey del Tahuantinsuyo, en la provincia de Chimborazo, acoge a 23 comunidades que se dedican al trabajo de la producción agrícola, como es el maíz la cebada, la papa, quinua frejol, crianza de ganados y artesanía, reciben a turistas y comparten con ellos sus costumbres, tradiciones y saberes ancestrales entre ellas la convivencia, elaboración de comida típica y artesanía, rituales tradicionales, explicación del trabajo en la agricultura, danzas, enseñándoles a los turista la vida en la zona urbana, permitiendo que estas actividades mejoren el desarrollo socioeconómico comunal.

La biodiversidad en la Amazonía es una región bendecida, miles de especies de fauna, flora acompasadas en ecosistemas de gran valía por sus complementos abióticos constitutivos 
estos son los componentes que le dan vida a la selva. Con el motivo de preservar los ecosistemas, se generan trabajos comunitarios para el aprovechamiento de los recursos naturales y culturales y mejorar la calidad de vida de los habitantes (Caiza Poma, I. R. 2015).

En la provincia de Pastaza se desarrolló un proyecto llamado Atacapi-Opip conformada por 132 comunidades indígenas, las cuales trabajan en la promoción y distribución de paquetes turísticos dirigidos a la ciudad de Puyo, entre las principales actividades que realizan son el turismo de aventura y turismo de naturaleza, la comunidad cuenta con todos los servicios básicos ofreciendo hospedaje en carpas, alimentación, transportes, e información y también excursiones hacia parques botánicos con una variedad de especies de flora y fauna propias de la región (Tamayo, G., Dávalos, X., \& Ponce, A. 2017).

El trabajo comunitario en la región litoral es un poco distinto a la región Amazónica y Andina, comunidades asociaciones costeras se reúnen para trabajar en actividades turísticas de la zona, como es la gastronomía, recorridos fluvial por recursos turísticos naturales, excursiones en diferentes sitios, proyectos comunales de balnearios, y pequeños pobladores que se dedican a la pesca. Entre ellos el Centro Turístico Comunitario Salango en el Parque Nacional Machalilla y Centro de Turismo Comunitario Boca de Gilces Crucita de la Provincia de Manabí (ENDERE, M. L., \& ZULAICA, M. L. 2015). (Rodríguez García, K. E. 2015).

La Coordinación zonal 5, está integrada por provincias tales como Guayas (excepto Guayaquil, Duran y Samborondón), Los Ríos, Santa Elena, Bolívar y Galápagos, incluye 48 cantones y 72 parroquias de la cual el $52 \%$ pertenece a la zona urbana y $48 \%$ zona rural (INEC, 2010), busca el incremento de ofertas de servicios turístico de calidad a nivel nacional y la participación de turismo en la economía. En referencia al trabajo comunitario se han generado distintos proyectos, principalmente en la provincia de Santa Elena donde existe indistintamente una gran cantidad de comunidades que actualmente basan sus actividades económicas en el turismo, como es el caso de la comunidad Sacachún, existen otros proyectos en la provincia de Guayas sistematizado en un programa llamado desarrollo comunitario que está basado en el mejoramiento de la calidad de vida, mediantes procesos metódicos, talleres, planificación, capacitación a grupos, fortaleciendo de acciones en el proceso de desarrollo auto sostenible. La provincia de Santa Elena a diferencia de la provincia de Guayas, su población mestiza se suman las etnias indígenas, basada en comunas, organizaciones sociales que trabajan en el rescate de la cultura local y trabajos comunitarios, la participación de las comunidades en las actividades turísticas permite la conservación y protección de los recursos y ecosistemas (Velásquez, G., \& Katherine, P. 2016). En la Provincia de Bolívar se encuentra la hilandería intercomunal de Salinas, una asociación de mujeres que trabajan en la elaboración de la artesanía y la tejedura como son bufandas chalinas, gorros y los más vendidos los famosos ovillos de lana. El negocio textilero comunitario cuanta con sus propias herramientas y mercados de comercialización cerca del centro de la ciudad de Salinas. (Tixe, Q., \& Rocio, M. 2018). 
Además se extiende al marco nacional, exportando a Estados Unidos, Alemania y Finlandia. La hilandería posee una sala de exposiciones donde muestran a los visitantes sus productos hechos a mano y fibra naturales, además muestran el proceso de elaboración a los turistas (Tixe, Q., \& Rocio, M. 2018).

Galápagos al ser un ecosistema frágil, existen medidas de protección, brigadas comunales trabajan en planes de manejo ambiental, capacidad de carga turística, áreas protegidas, conservación en la vida marina y silvestre con el objetivo de fortalecer la biodiversidad, en la provincia de Los Ríos, no se ha desarrollado formalmente el turismo comunitario sin embargo existen asociaciones agrícolas que están emprendiendo la actividad turística como fuente económica, como es el caso de la población del humedal abras de mantequillas en la ciudad de Vinces y la hacienda agroturística Luis y Cleo en la parroquia Febres Cordero, iniciativas que se complementan de la producción agrícola, la silvicultura, la pesca artesanal, la gastronomía y la avicultura, por otro lado Babahoyo y Quevedo son las ciudades que se dedican más al comercio artesanal e industria manufacturera. (Valverde Zurita, P. J. 2018)

La parroquia de Febres Cordero es una de las cuatro parroquias rurales pertenecientes al cantón Babahoyo, provincia de Los Ríos. Cuenta con 17.985 habitantes INEC, (2010) y su cabecera parroquial es el recinto Mata de Cacao.

El origen del nombre de dicha parroquia se dio en honor al ex presidente Venezolano Febres Cordero que en vista de su colaboración y preocupación por sus habitantes recibió el nombre de Febres Cordero, antecedente histórico que se dio en el año 1935.

La principal actividad económica de la parroquia es la producción agrícola, productos tales como el maíz, arroz y cacao, actividades pecuarias, turismo, comercio, artesanía, industrias. Cuenta con una extensión aproximadamente de $404 \mathrm{~km}$ cuadrados. La parroquia Febres Cordero del cantón Babahoyo posee sectores con potencialidades de interés turístico entre las que se menciona Balnearios de agua dulce en la Represa Rio Chico, Monserrate y Matilde Esther, La Junta Vieja, Junta Nueva, Represa Chilintomo, Pailón de San Jacinto y Providencia, San José de Febres Cordero. Sitios que lamentablemente no cuentan con apoyo para su desarrollo, encontrándose con infraestructura rudimentaria. (Plan De Desarrollo y Ordenamiento Territorial 2015-2019).

Las comunidades locales poseen recursos naturales para el desarrollo de las actividades turísticas pero coexiste una división en los habitantes los cuales se dedican a realizan otras funciones, abandonando el trabajo comunitario como iniciativa turística, sin pensar en los beneficios socioeconómico, aprovechamiento de la riqueza natural y mejoramiento de la calidad de vida que estas generan.

Además los recursos naturales de la Parroquia Febres Cordero no cuentan con mecanismo de control de seguridad turístico lo que impide que existan visitas de turistas con regularidad. 
La limitada accesibilidad es otro factor nocivo que impide el conocimiento de los recursos turísticos naturales, las entidades gubernamentales y otras, no apoyan al mejoramiento de infraestructura vial, comunidades local desconocen cómo tratar estos tipos de problema por el escaso asesoramiento en esta actividad turística.

La parroquia pide atención para promover el desarrollo integral en las comunidades, mitigando la pobreza (material) a través de la generación de empleo e ingresos económicos complementarios, evitando la migración de su población, fomentando una distribución justa de los ingresos y contribuyendo a la conservación del medio ambiente. (Inostroza, V.2008, p 90).

Mejora de la calidad de vida desde los organismos internacionales como la Organización Mundial de Turismo o la Organización Internacional Del Trabajo el turismo comunitario se asocia a los programas de lucha contra la pobreza. (Ballesteros, E. R., \& Carrión, D. S.2007, p100).

El trabajo comunitario se basa en una estrategia socio económica para los grupos comunitarios que se integran en apoyo mutuo, desarrollando actividades en beneficio de la colectividad, evitando la emigración desdés las comunidades y dando paso al turismo comunitario como fuente de empleo.

Según Moncayo, (2013) El turismo comunitario se caracteriza en que una porción considerable del control y los beneficios están en manos de miembros de comunidades locales. El Turismo Comunitario surge como una alternativa económica de las comunidades rurales, campesinas, indígenas, "mestizas" o afro-descendientes propias de un país, para generar ingresos complementarios a las actividades económicas diarias y defender y revalorizar los recursos culturales y naturales locales.

Henríquez, (2007) considera que el turismo comunitario es aquel que intenta contribuir con aquellas comunidades generalmente olvidadas por el modelo actual de desarrollo

El turismo comunitario se caracteriza por la integración de grupos tales como campesinas, indígenas, "mestizas" o afro-descendientes que buscan una trasformación en el desarrollo socioeconómico a través de la actividad turística en aprovechamiento racional de sus recursos.

Según la Revista Estado y Políticas Públicas (2015). Los recursos turístico naturales han sido y continúan siendo fuentes de crecimiento para la región de América Latina. Conforman la estructura productiva y podrían convertirse en la base de las actividades socioeconómicas, donde los países con mayor dotación lograrían importantes ventajas comparativas.

El concepto M.Andrea. (2009) Señala que recursos turístico naturales, al igual que la misma idea de "naturaleza", presenta una dificultad para ser pensados en la investigación social, ya que su misma etimología nos hace suponer un carácter "esencial" o inmutable. Pero los 
"recursos naturales" no son dados de manera objetiva, sino que se relacionan con necesidades sociales específicas.

Los recursos turístico naturales son bienes proporcionado por la naturaleza sin intervención de la actividad humana y son muy esenciales para el crecimiento turístico en una región, es indispensable relacionar los riesgos y problemas que con llevan a sostener éste recurso socio económico para las comunidades respecto a la disponibilidad de lograr satisfacer las necesidades futuras para el cuidado de nuestra naturaleza y transformando los recursos turísticos.

Según Palomeque, F. L. (2015) Los recursos turísticos son elementos que la naturaleza posee y sirven al ser humano al desarrollo socioeconómico y sostenible, es de suma importancia que estos recursos turísticos deben sean protegidos y conservado ya que la gran mayoría puede sufrir daños irreversibles.

Los recursos turísticos se dividen en naturales y culturales, al hablar de recurso turístico natural se refiere a la flora fauna y paisaje que proporciona la naturaleza sin la intervención del hombre por otro lado al hablar de recurso turístico cultura hace énfasis a las culturas, tradiciones y saberes ancestrales, donde intervine la mano del hombre.

Por medio de la pirámide de potencialidades turística se identifica los elementos que poseen un recurso turístico y proceder con su categorización.

Los recursos turísticos se dividen en naturales y culturales, recursos turísticos naturales tiene como elemento principal la flora, fauna y paisaje en cambio los recursos turístico cultural están conformados por tradiciones, costumbres y saberes ancestrales. Para que estos recursos turísticos se posesionen como atractivos deben satisfacer las 4 necesidades primarias del turista que son alimentación, hospedaje, información, trasporte, de la misma manera si el posicionamiento es de atractivo turístico se pone en juego la oferta y la demanda.

Al convertir un recurso en destino se toma en consideración las tendencias y mega tendencias turísticas a nivel mundial, al categorizar un destino turístico debe tener posicionamiento Internacional. En el caso de Ecuador el destino turístico es el Parque Nacional Galápagos. (Rivera, C. 2019).

La revisión del marco conceptual sustenta las posibilidades de integrar a la comunidad para el aprovechamiento de los recursos turísticos naturales, de acuerdo al Objetivo de Desarrollo Sostenible con el objetivo 8 (Trabajo Decente y Crecimiento Económico) ligado con el objetivo 12 (Producción y Consumo Responsable), esta investigación pretende mediante el desarrollo del trabajo comunitario estimular el crecimiento económico sostenible y la conservación de los recursos naturales.

PLANDETUR 2020 Objetivo 1 (Generar un proceso orientador que coordine los esfuerzos públicos, privados y comunitarios para el desarrollo del turismo sostenible, basado en sus 
territorios y bajo los principios de alivio a la pobreza, equidad, sostenibilidad, competitividad y gestión descentralizada). Por medio de este objetivo la presente investigación toma la iniciativa de integrar el trabajo comunitario en la actividad turística, generando beneficios económicos y fortalecimiento intercultural.

El presente trabajo toma referencia los EJES PLANDETUR 2020 que son instrumentos fundamentales para una equidad social y medioambiental. Gobernanza del Sistema turístico, Desarrollo de Destinos y Facilitación Turístico, Marketing y Promoción Turística, Formación y Capacitación de Recursos Humanos, Fomento de Inversiones y Gestión Financiera, Medidas Transversales, Gestión Socio Cultural y Ambiental para el Turismo.

La FEPTCE Federación Plurinacional de Turismo Comunitario del Ecuador, permite a la investigación tener un enfoque mucho más amplio sobre las iniciativas de turismo comunitario para mejorar la calidad de vida de las comunidades de nuestras nacionalidades y pueblos desde una perspectiva integral sustentable y sostenible con identidad, representándolas a nivel nacional e internacional permitiendo fortalecer y promover sus raíces.

Este proyecto de investigación se basa en el concepto de desarrollo según la Alianza para el Desarrollo Sostenible de Centroamérica (ALIDES) en el que se sustenta ésta investigación el trabajo comunitario es un proceso de transformación en la calidad de vida poblacional, encaminada al crecimiento económico con equilibrio y equidad social apoyándose en la actividad turística. Es el equilibrio ecológico del que implica el uso racional a la diversidad étnica y cultural regional, nacional y local. Vivir en un ambiente sano con, socio productivo sin comprometer y garantizando la calidad de vida de las generaciones futuras.

Objetivos específicos de la alianza para el desarrollo sostenible

- Elevar las tasas de crecimiento económico que permitan eliminar los niveles de pobreza y garantizar así la sostenibilidad social y política de los procesos de apertura económica y democratización de los países de la región.

- Promover, proteger y aprovechar en forma adecuada los patrimonios culturales y naturales.

- Mover una educación hacia el cuidado y aprovechamiento sostenible de los recursos naturales.

Estos objetivos permiten y apoyan a la investigación a integrar a la comunidad en trabajos comunitarios enfocados la actividad turística en consideración de los niveles de economía, la proteger los patrimonios naturales y cultural en base a la protección conservación sustentable.

\section{Metodología}

La metodología de esta investigación es Exploratorio, Descriptivo, Explicativo, porque pude observar cualidades que posee el objeto de estudio, las técnicas, instrumentos se aplicaran de acuerdo a los objetivos de la investigación. 
El método etnográfico y de observación permiten sistematizar expresiones sociales particulares que emergen por experiencias relatos, conversaciones que transcurren en la vida diaria de un individuo o en su conglomerado social, estas actividades son organizadas a través de técnicas de recolección de información como la encuesta y entrevista, cediendo a saberes ancestrales sobre un entorno en particular. Esta información imbrica sensaciones, emociones, hechos, valores o conductas expresadas por la población local. Esta investigación social se basó en parte, en información documental mundial y regional, para levantar el marco teórico sobre experiencias culturales y las necesidades turísticas básicas comunitarias, se elaboró diagnósticos participativos sobre las potencialidades poblacionales evidenciados la alegría de danzas folclóricas, juegos tradicionales, fiestas religiosas y los diferentes sabores gustativos.

\section{Resultados y Discusión}

Según los datos obtenidos de la muestra de 384 personas, se estableció el rango de edades entre 20 a 40 años, dando un resultado de 100 personas encuestadas. A través de las encuestas se concluye que existe un $100 \%$ de interés por parte de la comunidad de la parroquia Febres Cordero del cantón Babahoyo, provincia de Los Ríos, en desarrollar trabajo comunitario, están de acuerdo en integrar el recurso turístico natural Cascada pailón de San Jacinto, para realizar un circuito turístico en beneficio de la comunidad y aprovechamiento del mismo.

Tabla 1. Aprovechamiento sustancial del recurso hidrico.

\begin{tabular}{|l|c|l|}
\hline \multicolumn{1}{|c|}{$\begin{array}{c}\text { Aspectos conceptuales } \\
\text { importantes para la población }\end{array}$} & $\begin{array}{c}\text { Porcentaje de } \\
\text { aceptación } \\
\text { actitudinal }\end{array}$ & $\begin{array}{l}\text { Acciones procedimentales } \\
\text { en beneficio del } \\
\text { desarrollo territorial }\end{array}$ \\
\hline $\begin{array}{l}\text { El trabajo comunitario beneficia } \\
\text { la calidad de vida de la } \\
\text { comunidad }\end{array}$ & $100 \%$ & $\begin{array}{l}\text { Formar parte de la } \\
\text { Federación Plurinacional } \\
\text { de Turismo Comunitario } \\
\text { del Ecuador (FEPCE) }\end{array}$ \\
\hline $\begin{array}{l}\text { Considera usted, importante } \\
\text { integrar el recurso turistico } \\
\text { natural Cascada pailón de San } \\
\text { Jacinto para realizar un circuito } \\
\text { turístico en beneficio de la } \\
\text { comunidad }\end{array}$ & $99 \%$ & $\begin{array}{l}\text { Si } \\
\text { comunitario, están de } \\
\text { acuerdo en integrar el } \\
\text { recurso turístico natural } \\
\text { Cascada pailón de San } \\
\text { Jacinto, para realizar un } \\
\text { circuito turistico en } \\
\text { beneficio de la comunidad. }\end{array}$ \\
\hline
\end{tabular}

Fuente: Elaboración propia de los autores

La tabla anterior muestra evidencias sobre el comportamiento de la población frente a desafíos regionales, nacionales y mundiales, la idiosincrasia de nuestros pueblos es diluyente 
frente a las necesidades globales que exigen nuevas intervenciones comunitarias en integración de saberes ancestrales como carta de presentación turística frente al mundo cambiante, estos procesos turísticos como la asociatividad y la convivencia comunitaria, impulsan a conferir a las organizaciones plenamente reconocidas, iniciativas para implementar emprendimientos turísticos basado en el turismo comunitario que impera la realización de actividades comunes en mutuo aprovechamiento racional de recursos naturales y culturales prevaleciendo lo local en convivencia de acciones diarias compartidas con la legión de informales visitantes, temperando el accionar con manifestaciones lúdicas en su forma figurativa, fonética y simbólica. En la asociatividad, como norma de acción organizativa, prevalecen los intereses de la población en su conjunto y en la convivencia comunitaria como manifestación operativa prevalecen los intereses de los visitantes.

\section{Conclusiones.}

- La participación de la población en el trabajo comunitario es fundamental, la vinculación hacia el desarrollo sostenible, integra ámbitos de carácter grupal demostrado esto en la creación de redes participativas inclusivas, innovadoras y creativas líneas enmarcadas en la planificación nacional de formación y desarrollo de capacidades comunitarias mediante capacitaciones, talleres, asesoramiento técnico en busca de nuevos proyectos de interés mancomunado y asociativo con propuestas de gran valía sin interrumpir procesos empezados con anterioridad en aprovechamiento de los elementos endógenos y exógenos de la población rural.

- Construir comunidades competentes que refuercen sus indicadores de desarrollo con iniciativas y emprendimientos proyectistas es la misión del ministerio de turismo, la cámara provincial de turismo, la academia, entre otras instituciones que requieren diseñar en conjunto iniciativas de intervención comunitaria con polos opuestos de concentración de actividades alineadas en el Plan Nacional toda una vida.

\section{Referencias bibliográficas.}

- Acuña-Alfaro, J. (1999). Cultura del Desarrollo en Centroamérica: Evolución del Concepto de Desarrollo y su Influencia en la ALIDES. MSc. Tesis. Universidad Nacional. Heredia, Costa Rica.[www. geocities. com/jaacun/publications. html] \& Nowalski, Jorge (1998). Quién es Quién en la Institucionalidad Centroamericana: Algunas Reflexiones sobre el Proceso de Integración. PNUD-Proyecto CAM, 96.

- Rodríguez García, K. E. (2015). Oferta turística y el desarrollo socioeconómico de la comuna Las Gilces, Manglar La Boca, parroquia Crucita, cantón Portoviejo, Provincia de Manabí, República del Ecuador.

- Alcañiz, J. E. B., Simó, L. A., García, I. S., \& Herrera, A. A. (2008). Investigación internacional en marketing turístico: análisis de contenido sobre temas y metodologías. Comité Editorial Director: Agustín Santana Talavera, 6, 389.

- Ballesteros, E. R., \& Carrión, D. S. (2007). Turismo comunitario en Ecuador: desarrollo y sostenibilidad social. Editorial Abya Yala. 
- Bifani, P. (1999). Medio ambiente y desarrollo sostenible (No. 18). IEPALA Editoria

- Cabanilla, E. (2004). El Turismo Comunitario en el Ecuador: Situación y Tendencias Actuales FEPTCE, OIT, UCT.

- Cardona, M. N., \& Doria, R. B. (2015). El turismo comunitario en Colombia: iniciativa de desarrollo local y estrategia de empoderamiento del patrimonio cultural. Administración \&

- Cruz, C., \& Nicolás, M. (2016). Diseño de un programa de formación para el fortalecimiento

- del turismo comunitario de la comunidad de Mashpi, parroquia de Pacto del Distrito Metropolitano de Quito (Bachelor's thesis, Quito: Universidad de las Américas, 2016.). Desarrollo, 45(1), 129-141.

- Díaz, D. C., Hernández, I. P., \& Díaz, J. P. C. (2017). Implementación del turismo comunitario en función del desarrollo local. El caso del Consejo Popular de Sumidero/Implementation of Community Tourism Based on Local Development. The Case of the Sumidero Popular Council. Estudios del Desarrollo Social: Cuba y América Latina, 5(1), 27-36.

- Escolar, B. M., \& Morueco, R. F. (2011). Vino, turismo e innovación: las Rutas del Vino de España, una estrategia integrada de desarrollo rural. Estudios de economía aplicada, 29(1), 5-35.

- Fortunato, N. (2005). El territorio y sus representaciones como recurso turístico: Valores fundacionales del concepto de" parque nacional". Estudios y perspectivas en turismo, 14(4), 314-348.

- Gallopín, G. C. (2003). Sostenibilidad y desarrollo sostenible: un enfoque sistémico. CEPAL.

- Gómez Schettini, M., Almirón, A., \& González Bracco, M. (2011). La cultura como recurso turistico de las ciudades: El caso de la patrimonialización del tango en Buenos Aires, Argentina. Estudios y perspectivas en turismo, 20(5), 1027-1046.

- Gonzáles, L. M. C. (2017). Desarrollo económico local: leyendas y realidades. Territorios, (1), 9-24.

- Guzmán, T. J. L. G., \& Cañizares, S. M. S. (2009). Turismo comunitario y generación de riqueza en países en vías de desarrollo. Un estudio de caso en El Salvador. REVESCO. Revista de Estudios Cooperativos, (99), 85-103.

- Henríquez, C., Zechner, T. C., \& Sampaio, C. A. C. (2017). Turismo y sus interacciones en las transformaciones del espacio rural. Revista Austral de Ciencias Sociales, (18), 21-31.

- Herrero, L. M. J. (2017). Desarrollo sostenible. Ediciones Pirámide.

- Inostroza, V. (2008). Aportes para un modelo de gestión sostenible del turismo comunitario en la región andina. Gestión Turística, (10).

- Jiménez Herrero, L. M. (1997). Desarrollo sostenible y economía ecológica.

- M.Andrea..Ambiente \& Sociedade Campinas v. XII, n. 2. p. 405-414. jul.-dez. 2009

- Morgado, H. F. M. (2006). Turismo comunitario: una nueva alternativa de desarrollo indígena. AIBR, Revista de Antropología Iberoamericana, 1(2), 228-243.

- Velásquez, G., \& Katherine, P. (2016). Propuesta de políticas que definan y guíen la comunicación institucional con comunidades del sector rural del país, tomando como 
referencia la población afiliada al seguro social campesino (Master's thesis, Quito: UCE).

- Naval, V. P., Serra, J. P., \& Mangana, R. (2017). Desarrollo local y turismo. El impacto socioeconómico de la comunicación digital en Portugal. Revista Latina de Comunicación Social, (72), 1515-1535.

- Tixe, Q., \& Rocio, M. (2018). Diseño de un modelo de gestión de calidad en la empresa FUNORSAL de la provincia de Bolívar (Bachelor's thesis, Universidad Técnica de Ambato. Facultad de Ciencias Administrativas. Carrera Organización de Empresas).

- Navarro, D. (2015). Recursos turísticos y atractivos turísticos: conceptualización, clasificación y valoración. Cuadernos de turismo, (35), 335-357.

- Palomeque, F. L. (2015). Modalidades turísticas y tipológicas de espacios turísticos. Papers de Turisme, (11), 49-64.

- Pastor, V. J., Jurado, C. C., \& Soler, D. A. (2018). Desarrollo rural a través del turismo comunitario. Análisis del valle y cañón de Colca. Gestión turística, (15), 120.

- Pérez, M. M. (2018). El desarrollo local sostenible. Revista Economía y Desarrollo (Impresa), 140(2).

- Revista Estado y Políticas Públicas No 5. Año 2015. ISSN 2310-550X pp. 99-118

- Rivera García, C. Oviedo Rodríguez, M. Galarza Bravo F. (Abril 2016) Visión y Planeación Turística; Responsabilidad 100\% Humana (Pag. 20-29,84,130).

- Ruiz, E., Hernández, M., Coca, A., Cantero, P., \& Campo, A. D. (2008). Turismo comunitario en Ecuador. Comprendiendo el community-based tourism desde la comunidad. Pasos. Revista de turismo y patrimonio cultural, 6(3).

- Sachs, J. D., \& Vernis, R. V. (2015). La era del desarrollo sostenible. Deusto.

- Fayos-Solá, E. (2004). Política turística en la era de la globalización. Mediterráneo económico, 5.

- Díaz, D. C., Hernández, I. P., \& Díaz, J. P. C. (2017). Implementación del turismo comunitario en función del desarrollo local. El caso del Consejo Popular de Sumidero/Implementation of Community Tourism Based on Local Development. The Case of the Sumidero Popular Council. Estudios del Desarrollo Social: Cuba y América Latina, 5(1), 27-36.

- Frías, C. C. H. R. (2018). La economía solidaria para el desarrollo del turismo comunitario en la Isla Santay. Universidad y Sociedad, 10(3), 159-164.

- Pochat, V., Donoso, M., \& Saldarriaga, J. (2018). Proceso Regional de las Américas: Foro Mundial del Agua 2018: Informe subregional América del Sur: Resumen ejecutivo.

- Solís Ruiz, M. A. (2016). Aculturiación de saberes ancestrales y la vulnerabilidad de la dinámica familiar en la etnia indígena Salasaca (Master's thesis, Universidad Técnica de Ambato, Facultad de Jurisprudencia y Ciencias Sociales, Maestría en Trabajo Social familiar).

- Caiza Poma, I. R. (2015). Plan estratégico turístico de los valores patrimoniales del cantón Colta en la provincia de Chimborazo (Bachelor's thesis, Quito: UCE). 
- Tamayo, G., Dávalos, X., \& Ponce, A. (2017). Análisis de competitividad aplicado a las empresas del sector de turístico de la amazonía ecuatoriana. UTCIENCIA, 2(2), 96-108.

- Rodríguez García, K. E. (2015). Oferta turística y el desarrollo socioeconómico de la comuna Las Gilces, Manglar La Boca, parroquia Crucita, cantón Portoviejo, Provincia de Manabí, República del Ecuador.

- ENDERE, M. L., \& ZULAICA, M. L. (2015). Sustentabilidad Socio-cultural y Buen Vivir en Sitios Patrimoniales: Evaluación del caso Agua Blanca, Ecuador. Ambiente \& Sociedade, 18(4), 265-290.

- Valverde Zurita, P. J. (2018). Turismo comunitario y la identidad cultural de los habitantes del recinto el recuerdo en el humedal internacional abras de mantequilla, cantón Vinces, provincia de los Ríos (Bachelor's thesis, Babahoyo: UTB, 2018).

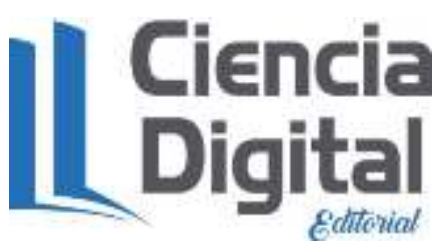


Para citar el artículo indexado.

Rivera García, C., Arellano Valencia, D., \& Hurtado, S. (2019). Trabajo comunitario en el recurso turístico natural cascada Pailón San Jacinto, Parroquia Febres Cordero, Cantón Babahoyo. Explorador Digital, 3(3), 62-76. https://doi.org/10.33262/exploradordigital.v3i3.693

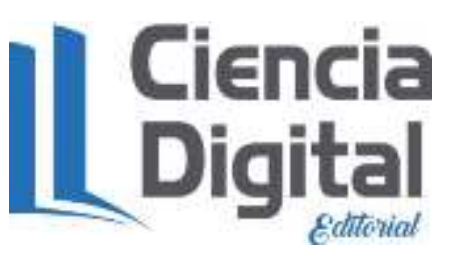

El artículo que se publica es de exclusiva responsabilidad de los autores y no necesariamente reflejan el pensamiento de la Revista Explorador Digital.

El articulo queda en propiedad de la revista y, por tanto, su publicación parcial y/o total en otro medio tiene que ser autorizado por el director o editor de la Revista Explorador Digital.
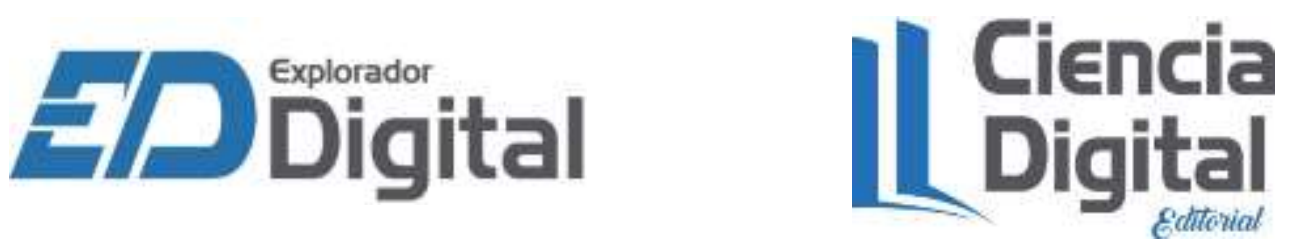